\title{
The Mosquito Hypothetically Considered as the Transmitting Agent of Yellow Fever
}

\author{
Carlos J. Finlay MD
}

This essay, now in the public domain, is a translation of Finlay's historic speech on August 14, 1881, to the Royal Academy of Medical, Physical and Natural Sciences in Havana, Cuba. Excerpted from Jose López Sánchez. Carlos J. Finlay: His Life and His Work. Instituto Cubano del Libro. Editorial José Martí, 1999.

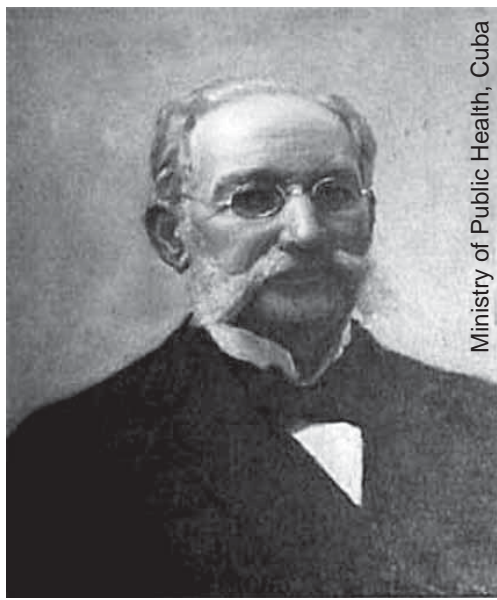

Some years ago, in this same place, I was honored to expound the result of my alkalimetric tests with which I believed [translation error corrected-Eds.] to have definitely proved the excessive alkalinity of the atmosphere in Havana. Perhaps some of the Academicians present remember the alleged relations I sought to point out between that fact and the development of yellow fever in Cuba. But much has been done since then, more exact data has been collected, and the etiology of yellow fever has been more methodically studied than in previous times. I became, therefore, convinced that any theory that attributes the origin or propagation of that disease to atmospheric, miasmatic or meteorological influences, to uncleanliness or to the neglect of general sanitary measures, is absolutely untenable. I have left my original beliefs behind; and by saying this here, I want to justify this change of mind by submitting to the judgement of my distinguished colleagues a new series of experimental studies I undertook in order to find out how yellow fever spreads.

I must warn you, however, that this work has nothing to do with the nature or the form in which the cause of yellow fever may exist: I am just acknowledging the existence of a material, transportable cause that may be an amorphous virus, an animal or vegetable germ, a bacteria, etc., but that in any case is something tangible that has to be conducted from a sick person to a healthy person for the disease to spread. What I purport to study is the means by which the matter that causes yellow fever detaches itself from the body of a sick person and introduces itself into a healthy person. The need to acknowledge an agent extraneous to the disease so that it is transmitted is the consequence of numerous considerations, some of which were already stated by Rush and Humboldt at the beginning of this century, and were later confirmed by more recent observations.

Yellow fever sometimes crosses the ocean and propagates in distant cities that boast quite different meteorological conditions from the center from which the infection stemmed; while, at other times, the same disease does not spread beyond a narrow epidemic area, irrespective of the fact that the meteorology and the topography of the surrounding areas show no differences that explain the distinct behavior of the same disease in two apparently similar sites. Once the necessary interference of a transmitting agent that explains those anomalies is acknowledged, it becomes clear that the influence of all conditions recognized as essential for yellow fever to spread must befall that agent. It was, therefore, impossible to look for that agent among microorganisms or zoophyte, because meteorological fluctuations that most affect the development of yellow fever exert little or no influence over these lowest categories of animate nature.

To fulfill this first requisite, it was necessary to seek among insects and, bearing in mind that yellow fever is medically and alsoaccording to recent works-histologically characterized by vascular lesions and physicochemical alterations of the blood, it seemed natural to look for an insect that could carry infectious particles from a sick man to a healthy man, among the ones who pierce blood vessels to suck human blood. I finally asked myself, after considerations it is not necessary to mention here, if the mosquito could be the transmitter of yellow fever. This was the hypothesis behind the series of experimental studies I will expound.[...]

It is true that the mosquito exists in all latitudes, but it is not equally abundant on every site. During their travels across equinoctial America, Alexander Humboldt and Bonpland said, "The torment of mosquitos is not as widespread under the torrid zone as it is generally believed. They are not more abundant on the high plateaus that rise more than 400 toises over the sea level, or on the dry plains removed from the great rivers, like the Cumaná and the Calabozo, than in the most populated places in Europe." The influence of dry weather conditions and the distance from rivers, which those travelers pointed out, is understandable, for the larva of the mosquito and its pupa are aquatic and, in order to propagate, the adult insect has to oviposit on the water. In regard to the hindrance heights pose to its propagation, I think it is a consequence of the difficulty these dipteron always experience in upward flight after gorging with bloodparticularly in the case of the Culex mosquito, whose wings are very small—for this difficulty only increases due to the rarefaction of the air at great heights. In such cases, it is understandable that the mosquito instinctively avoids these places. Said travelers also say the missionary Bernardo Zea had built a room on a platform made from palm tree trunks, where they used to go in the evening to dry the plants they had collected and make notes in their diaries. "The missionary had rightly observed," they say, "that insects usually abound on the lower layer of the atmosphere, which is 12 to 15 feet above the ground level." And they add, "as one climbs toward the Andean plain or plateau, these insects disappear and pure air can be breathed there... mosquitos are not to be feared at a height of 200 toises."

The mosquito has historically been one of the insects observed since olden times. Aristotle and Pliny refer to its proboscis, used 
to pierce the skin and suck the blood. The Greek historian Pausanias (quoted by Taschenberg) mentions the city of Myus, in Asia Minor, located on a cove whose connection with the sea was later cut off; when the water of the lake thus formed was no longer salty, there was such a plague of mosquitos that the city's inhabitants left it and moved to Miletus. We can also read in Herrera's Decades that when Juan de Grijalva first discovered the coasts of New Spain in the year 1518, he and his people occupied an islet he named San Juan de Ulúa and had to build their huts "on the highest dunes on the islet to flee from the annoyance of the mosquitos." They had to leave that place after seven days, for "they could not get rid of the mosquitos," and Bernal Díaz del Castillo had to leave for certain Indian temples "fleeing from the bothersome mosquitos." Lastly, in 1519, in almost the same place where the modern Veracruz is located nowadays, "long-legged mosquitos," says Herrera, "and the small ones which are even worse, vexed Cortés' men."[...]

It is known that only the female mosquito bites and sucks blood, and that the male feeds on vegetable juices, mainly the sweet ones, but to date I have not found in any of the authors who have written about this matter that the female does not bite before having been impregnated by the male. This, at least, is what could be inferred from the following experiments: a female of the Culex mosquito, trapped as it left the pupa and kept alive for two or three days, could not be prompted to bite. I have repeated this experiment several times with the same negative result.

Females trapped during impregnation, bite and sate themselves with blood as soon as the mating is completed.

In short, almost all females trapped after feeding oviposit a few days later, while the impregnated ones which are unable to feed, die without having oviposited.

Therefore, the female mosquito is not avid to feed on live blood to sustain itself, and, in fact, it would be difficult to understand why such an enormous quantity of a rich nourishment like pure blood is necessary to sustain such a diminutive body. Hence, I was obliged to admit that blood was destined for other purposes related to the propagation of the species. I am inclined to surmise, as the most natural among my hypothesis, that the influence of the blood is due to its temperature because, in order to mature, the ovules contained in the ovaries of the female mosquito require a temperature of $37{ }^{\circ} \mathrm{C}$, which would difficultly be obtained with such certainty in our island's meteorological conditions, but for the means used by the mosquito, which sucks a considerable quantity of blood at the required temperature; perhaps, at a given time and for its own ends, the mosquito chooses a feverish person whose blood at $39{ }^{\circ} \mathrm{C}$ or $40{ }^{\circ} \mathrm{C}$ accelerates the time to oviposit. Thus, it also becomes clear why the long-legged mosquito and other large mosquitos can absorb at once all the blood needed to mature with its heat from 200 to 350 eggs which they in fact oviposit in a single laying, while the smaller species, like the Culex mosquito, need to suck their fill of blood several times in order to begin laying and usually oviposit two or three times.

Once the female mosquito is sated, it needs two, three and even four days, according to the species, to digest the blood, during which time, hidden from indiscreet eyes, it spends long hours in strange maneuvers Reaumur was unable to explain, only because he observed them when performed by mosquitos in their natural environment. When they are trapped in glass tubes, it is easy to realize those movements are aimed at covering the entire body with a sticky secretion the mosquito gathers from its anus with its hind paw. Using those paws, it covers the entire body: each paw separately, the abdomen, the wings, the thorax, the head, and the proboscis itself. As our distinguished academician Don Felipe Poey suggested, this operation may be aimed at rendering the female mosquito impregnable for the moment it oviposits on the water. The mosquito defecates bloody particles while it digests the blood; these particles dissolve in the water quite easily, even after having been dry for several months. This is undoubtedly due to the combination of the blood and the saliva the insect pours into the wound destined, according to a generalized opinion, to render the blood it sucks more fluid. Usually, after sucking all the blood of an uninterrupted bite, the mosquito does not bite again but, on the contrary, avoids alighting on the naked skin (undoubtedly because it dislikes its warmth) until it has digested all the blood. This is the time in which the long-legged mosquito oviposits.[...]

It is evident that, according to my viewpoint about the mosquito, the species Culex mosquito is admirably endowed to carry a disease transmissible from one individual to another by means of the blood, for it has multiple occasions to suck blood from different subjects and also to infect various individuals, thus noticeably increasing the probabilities that its bite has the necessary requisites to carry out the transmission. On the other hand, when the Culex cubensis absorbs through its proboscis a larger quantity of virulent blood, it will become more impregnated and able to produce a more serious inoculation, mainly if it is carried out shortly after the long-legged's lancets have left the capillary vessel of a sick person, as it must happen when its first bite has been interrupted. In this case, therefore, the infection will be more serious but its occurrence will be less probable.[...]

It is known that, although they never disappear altogether in Havana, mosquitos are more numerous in certain seasons than in others. It seems to me their numbers increase from April or May until August, decreasing gradually until February and March. But there is a point related to the study we have been carrying out that should not be overlooked for the numerous applications it may have in certain - to date unexplained-outbreaks of yellow fever, without new importation, on sites until then considered immune. I am referring to the mosquito's hibernating period, a phenomenon not observed in our clime, at least in all its stages, which is, according to authorized criteria, the most usual way in which the species propagates in cold climes. Dr Taschenberg says that "impregnated females of the last generation hibernate in the most diverse hideouts, mainly in household attics, in order to propagate the species on the following spring."

In regard to conditions that favor the development of mosquitos, I will mention heat, dampness, stagnant water, low, dark sites, the absence of wind, and summertime; but we should not omit Humboldt's observation about the fact that the abundance of mosquitos is not always due to fixed meteorological or topographic conditions. I have already referred to the difficulties the mosquito must experience, due to its relatively short wings, to rise in the air after having fed. The same cause will prevent the mosquito from moving too far away from the place where it bit for the last time and, in general, from keeping itself in the air for a long time or travel great distances without alighting. But this does not prevent the mosquito, hidden among the clothes, in a hat, in a suitcase, 
etc., after having recently fed, from being transported long distances perhaps carrying in its lancets the inoculable germ of the disease.[...]

After this lengthy but necessary explanation about the habits of our mosquitos in Cuba, and specifically the Culex mosquito, let us see what means the mosquito can avail of to transmit yellow fever, if this disease were truly transmissible by inoculating the blood. The most natural thing regarding this question is to think that the virulent blood the mosquito has sucked from a person afflicted with yellow fever, which can amount from five to seven or eight cubic millimeters, the same quantity that, were the mosquito to die before having digested it, would still be in excellent conditions to keep its infectious properties for a long time. One could also think, undoubtedly, about the same blood the mosquito deposes as excrement on drinking and other waters, which could very well carry the infection if it were susceptible to introduce itself through the mouth. But Firth's experiments and certain considerations linked directly to my way of viewing the pathogeny of yellow fever did not allow me to halt on any of those means of propagation. I will explain why.

When the members of the North American Commission on Yellow Fever said farewell to us two years ago, they left us their valuable collection of photographs of the microscopic preparations made by our corresponding associate Dr Sternberg; my attention was drawn to the fact that they showed the red corpuscles of the blood remained whole in yellow fever hemorrhages; and as those hemorrhages sometimes take place without any appreciable rupture of the vessels, we were obliged to deduce that, that being the most essential clinical symptom of the disease, the main lesion would have to be sought in the vascular endothelium. The first stage would be the invasive fever, remission would coincide with the eruptive stage, and desquamation would be the third stage. If it occurs on favorable conditions, the sick person would only show traces of an exaggerated filtration of some elements of the blood through the new endothelium; if it took place on unfavorable conditions, the endothelium, not having fully recovered, would not be able to prevent the exit of the elements which appear in the blood, passive hemorrhages will ensue, and the patient will be in imminent danger. In short, comparing this disease to smallpox and to the vaccine, I told myself that, in order to inoculate it, the inoculable matter would have to be sought inside the vessels of a person afflicted by yellow fever and taken into a blood vessel of another person capable of being inoculated. The mosquito fulfills all these requirements extremely well with its bite, which we could never imitate with the comparatively crude and coarse tools even the most skilled of our artisans can produce.

Therefore, three requisites would be necessary for yellow fever to propagate: (1) the existence of a person afflicted with yellow fever, whose capillaries the mosquito can pierce with its lancets and impregnate them with virulence particles, during the adequate stage of development of the disease; (2) to prolong the mosquito's life between the time it bit the sick person and the time in which it should reproduce the disease; and (3) the subject the mosquito bites after that is someone able to contract the disease.

It can be ascertained that the first of these requisites has always been fulfilled in Havana, since Dr. Ambrosio G. del Valle began to publish his valuable bulletin on fatalities; in regard to the second and the third requisites, it is evident that the probabilities for their fulfillment will depend on the abundance of mosquitos and the number of individuals susceptible of being inoculated in a given site. In fact, I think these three requisites have always coincided in Havana in the years during which yellow fever has wreaked greater havoc.

Such is my theory, gentlemen, and it has been particularly strengthened by the numerous historic, geographic, ethnological and meteorological coincidences found among the available data about the mosquito and about yellow fever, and also by the fact that we can assist ourselves of them to explain circumstances unexplained to date by existing theories. Yellow fever was unknown to the white race until after the discovery of America and, according to Humboldt, it is a traditional criteria in Veracruz that the disease has existed there since the first Spanish explorers landed on its shores. We have also verified that the Spanish, ever since their first voyage, referred to the presence of mosquitos, more evident in the sand dunes at San Juan de Ulúa than in any other place in America. The races most prone to be afflicted by yellow fever are also the ones that suffer the most from mosquito bites.

The most favorable meteorological conditions for the development of yellow fever are the same that increase the number of mosquitos; to support that assertion, I can refer to various partial epidemics about which it is said-and competent physicians also avow it-that, during the prevalence of yellow fever, mosquitos had been much more numerous than before; it was verified in one of those cases that the mosquitos belonged to a different species than the ones usually observed in that given site, and that there were gray spots on their bodies. In regard to the topography of yellow fever, Humboldt himself, who pointed out the height mosquitos are able to reach, elsewhere mentions the highest altitude to which yellow fever can propagate. And lastly, the well known case of the North American steamship Plymouth-where two cases of yellow fever developed on high seas, after the ship had been disinfected and frozen during the whole winter season, and after four months had elapsed since the last case had occurred aboard in the previous November-can be fully explained by the period of hibernation of the mosquitos that bit the previous cases of black vomit that, under tropical conditions, came out of their lethargy and bit two of the new members of the ship's crew.

Thus supported by these reasons, I decided to test my theory and, after obtaining due authorization, I proceeded as follows.

Last June 28, I took a mosquito, trapped before it had bit anyone, to Garcini's Health House, and I prompted it to bite and sate itself with the blood of a sick man, Camilo Anca, a perfectly characterized five-day case of yellow fever, who died of that disease two days later. I then chose F.B., one of twenty-one healthy individuals not acclimated to the disease who are currently under my observation, and had that same mosquito bite him on June 30 . Bearing in mind that the incubation of yellow fever, as verified in some special cases, fluctuates between one and fifteen days, I continued to observe the aforementioned F.B. He began to feel sick on the ninth, and on the fourteenth he was admitted to the hospital, suffering from a benign case of yellow fever perfectly characterized, however, by the icterus and the presence of albumin in the urine, which persisted from the third day until the ninth day.

On July 16 , I had a mosquito bite Domingo Rodríguez, a serious case of yellow fever confined in Garcini's Health House, who had 
been sick for three or four days. On the twentieth, I had the same mosquito bite me and, lastly, on the twenty-second, I made it bite A.L.C., another one of the twenty persons under observation. Five days later, he was admitted to the hospital suffering from fever, strong headaches and waist pains, and flushed face; these symptoms persisted for three days, and the patient began to convalesce without having presented any icterus or albuminuria. The physician in his care diagnosed aborted yellow fever.

On July 29 , I had a mosquito bite D.L.R., another serious threeday case of yellow fever at Garcini's Health House. On the thirtyfirst, I had it bite [translation error corrected-Eds.] D.L.F., another of the twenty individuals I was observing. On August 5, at two o'clock in the morning, he presented the symptoms of a slight case of yellow fever; he later presented an icterus, but I believe no trace of albumin was found; in any case, his disease was classified as aborted yellow fever.

Finally, on July 31, I had another mosquito bite the same D.L.R., confined in Garcini's Health House, on his fifth day of the disease from which he died on the following day. On August 2, I had that same mosquito bite D.G.B., another one of my twenty patients. To date, his last inoculation has produced no results, but as only twelve days have elapsed, it is still within the limits of the incubation period.*

I must warn you that the persons I have just mentioned are the only ones whom I have inoculated with a mosquito in the way I have indicated, and that since June 22 to date (a full seven weeks) no further confirmed cases of yellow fever or of aborted yellow fever have occurred among the twenty people under my observation, save the first three that were inoculated.

These tests certainly favor my theory, but I do not wish to exaggerate in considering as fully verified something which still needs further proof, however many the probabilities I can invoke in my support. I understand fully well that no less than an irrefutable demonstration is necessary for a theory that essentially dissents from the ideas propagated to date about yellow fever to be accepted. But while the data we still lack becomes available, allow me to summarize the most essential points I have endeavored to prove in the following conclusions.

\section{CONCLUSIONS}

1. It has been confirmed that the Culex mosquito usually bites several times during his life, not only when its first bite has been accidentally interrupted but also when it has been able to sate itself; in the latter case, two or three days elapse between bites.

2. As the mosquito's lancets are placed in such a way as to preserve the particles suspended in the liquids on which the insect feeds, the possibility cannot be denied that a mosquito keeps in its lancets particles of the virus contained in infected blood and inoculates it to the people it successively bites.

3. Direct tests to determine whether the mosquito can transmit yellow fever in such a way have been limited to five attempts at inoculation by a single bite, whose results were: one case of benign yellow fever, perfectly characterized, however, by both albuminuria and icterus; two cases classified by attending physicians as aborted yellow fever; and two cases of slight, ephemeral fever of undefined nature. ${ }^{*}$ From which it can be inferred that the inoculation by a single bite is not enough to produce yellow fever in its gravest forms; an assessment regarding the efficacy of the inoculation was therefore postponed until it is possible to carry out the experiment under entirely conclusive circumstances, that is to say, outside the epidemic area.

4. If it were verified that the inoculation by the mosquito can reproduce yellow fever and is also the general means through which the disease propagates, the conditions in which that dipteron lives and develops would explain the anomalies pointed out to date regarding the spread of yellow fever, and we would have the means, on the one hand, to prevent the disease from spreading and, on the other, to safeguard individuals prone to contract it by means of a benign inoculation. My sole intention is that note be taken about my observations and that the task of evincing what truth there is in my concepts be left to direct experiments. This does not mean, however, I am avoiding a debate on the ideas I have expounded; quite on the contrary, I will be more than pleased to heed the warnings or objections my distinguished colleagues wish to put forward. -1 -

* The patient D.G.B. came to be examined on August 17, and said he had been suffering from headaches, lack of appetite and general indisposition for six days. On the twenty-fourth, he was running some fever (pulse 100; temperature $38{ }^{\circ} \mathrm{C}$ ), and said the fever had been higher on the eve and during the morning of that same day. However, it was only a slight fever; the patient did not die nor did he need any medication. The fever stopped, but the headache continued for a few days more.

** I.C., one of the twenty individuals, was bitten on August 15 by a mosquito that had previously bitten a patient at the Military Hospital on his fifth day of sickness. The individual thus inoculated does not seem to have developed the disease to date (September 1). I have not been able to see him after the inoculation, and only by word of mouth was I advised that he felt somewhat ill on August 24 and 25, but had not died either. 